\title{
Instituto Mesoamericano de Salud Pública: órgano de apoyo técnico y fortalecimiento del Sistema Mesoamericano de Salud Pública
}

\author{
Miguel A González-Block, D Sc,(I) Juan Francisco Molina-Rodríguez, MMS,(') Víctor Becerril, M en Soc,(') \\ Emily Vargas, Mtra, ${ }^{(I)}$ Consejo Directivo del IMSP**
}

\begin{abstract}
González-Block MA, Molina-Rodríguez JF, Becerril V, Vargas E, Consejo Directivo del IMSP. Instituto Mesoamericano de Salud Pública: órgano de apoyo técnico y fortalecimiento del Sistema Mesoamericano de Salud Pública. Salud Publica Mex 20II;53 supl 3:S358-S367.
\end{abstract}

\section{Resumen}

Objetivo. El Instituto Mesoamericano de Salud Pública (IMSP) se constituyó en 2009 como órgano técnico del Sistema Mesoamericano de Salud Pública (SMSP) y la RedVirtual de Instituciones Académicas. En la primera fase se identificaron las necesidades de fortalecimiento de sistemas de salud y se evaluaron los primeros resultados de capacitación. Material y métodos. Se realizó un análisis de contenido de los $\mathrm{Pla}$ nes Maestros del SMSP para cada prioridad y se encuestó a los integrantes del Grupo de Trabajo en Malaria y Dengue. Resultados. Se identificaron los temas de capacitación requeridos por áreas de prioridad del SMSP y las necesidades de gestión de conocimiento para control y eliminación de la malaria y dengue. Se elaboró un mapeo de competencias a desarrollar con el personal estratégico, táctico y operativo. El IMSP capacitó a 9I funcionarios de ocho países en su primer año. Estas actividades se desarrollaron de julio 2009 a junio 2010 , en consulta a directivos de servicios de salud de países integrantes del Sistema Mesoamericano de Salud Pública, Colombia y México. Conclusiones. El IMSP está respondiendo a las necesidades de salud pública en la región mesoamericana.

Palabras clave: regionalización; América Latina; educación; gestión del conocimiento
González-Block MA, Molina-Rodríguez JF, Becerril V, Vargas E, Consejo Directivo del IMSP.

Mesoamerican Public Health Institute:

Technical support and capacity strengthening arm

of the Mesoamerican Public Health System.

Salud Publica Mex 20I I;53 suppl 3:S358-S367.

\section{Abstract}

Objective. The Mesoamerican Public Health Institute (IMSP) was constituted in 2009 as the technical organ of the Mesoamerican Public Health System (SMSP) and the Virtual Network of Academic Institutions. Health system capacity strengthening needs and preliminary training results were assessed in the first phase. Material and Methods. The SMSP Master Plans were content-analyzed for each priority and members of the Malaria and Dengue Working Group were surveyed. Results. The training needs required for each SMSP priority area were identified and knowledge management needs for malaria and dengue analyzed. Competencies were mapped across strategic, tactical and operative personnel that will be requiring them. IMSP trained in its first year 91 persons in eight countries. Conclusions. IMSP is responding to the Mesoamerican region's public health needs.

Key words: regional health planning; Latin America; education; knowledge management * Miguel Orozco-Valladares (presidente),Arturo Barrio-González, Rolando Hernández, Jaime Más-Oliva, Javier Nieto, Mario Henry-Rodríguez, Esperanza Tuñón,
Fátima Valle-de Zúñiga, Luis Bernardo Villalobos.

(I) Centro de Investigación en Sistemas de Salud, Instituto Nacional de Salud Pública. Cuernavaca, Morelos, México.

Fecha de recibido: 12 de enero de 20II - Fecha de aceptado: 7 de junio de $201 \mathrm{I}$

Autor de correspondencia: Mtro. Juan Francisco Molina-Rodríguez. Centro de Investigación en Sistemas de Salud. Instituto Nacional de Salud Pública. Av. Universidad 655, col. Santa María Ahuacatitlán. 62100 Cuernavaca, Morelos,

Correo electrónico: francisco.molina@insp.mx 
E Proyecto Mesoamérica de Integración y Desarrollo busca dar un renovado esfuerzo al tema de salud, en el ámbito de desarrollo social, a través del Sistema Mesoamericano de Salud Pública (SMSP). El SMPS es la plataforma para dar respuesta eficaz y oportuna a las necesidades de salud en la región. Funciona como un mecanismo de coordinación y desarrollo de actividades de cooperación multilateral, que busca consolidar la cooperación técnica horizontal entre los países de la región. El SMSP busca complementar las iniciativas que ya operan en el marco del Consejo de Ministros de Salud de Centroamérica (Comisca).

El SMSP es innovador por su escala regional, por la participación de actores globales tanto gubernamentales como privados en el financiamiento y el apoyo técnico y por su claro enfoque en los Objetivos del Milenio 3, 4 y 5 . Para su implementación se requiere del fortalecimiento de los sistemas nacionales de salud y de los mecanismos de coordinación regional. ${ }^{1}$ Es de esperarse que las inversiones que acompañen a las intervenciones implementadas por el SMSP contribuyan también al fortalecimiento de los sistemas nacionales de salud bajo un enfoque diagonal. ${ }^{2}$

El personal estratégico encargado de la implementación del SMSP debe contar con las competencias para responder: ¿qué está pasando en el diseño, implementación, administración, operación, servicios y resultados de las intervenciones del SMSP?, ¿es lo que se espera o se desea?, y ¿por qué está sucediendo de esa forma? ${ }^{3}$ Las competencias habrán de incluir la capacidad para analizar las intervenciones y estrategias de control de enfermedades, tanto en sus elementos comunes en el ámbito regional, como en aquellos propios de los ámbitos nacional y local. Las innovaciones habrán de ser integradas a los sistemas nacionales para lograr el fortalecimiento mutuo. Asimismo los funcionarios habrán de reconocer las fortalezas y debilidades de los sistemas de salud, así como las oportunidades y amenazas en el contexto económico, político y cultural. Los funcionarios deberán contar también con las competencias para gestionar un proceso de cambio activo destinado a alcanzar la adopción individual y organizacional del SMSP. ${ }^{4}$

Se cuenta ya con muchas herramientas para promover la implementación sustentada en la mejor evidencia, ${ }^{5-8}$ incluyendo metodologías y mecanismos de interacción para aprovechar tanto la experiencia y el juicio de los decisores como el conocimiento derivado de la investigación. ${ }^{7,9,10}$ Los conceptos de transferencia y gestión del conocimiento se encuentran entre las metodologías más utilizadas para identificar, analizar, evaluar, organizar y compartir el conocimiento científico para sustentar la toma de decisiones. ${ }^{10,11}$ Diversas organizaciones internacionales han desarrollado herra- mientas destinadas a apoyar este proceso. ${ }^{12-14}$ En México, existen experiencias de establecimiento de interfaces entre la oferta de los investigadores con la demanda de conocimiento pertinente originada entre los decisores. ${ }^{15-17}$ Destaca la creación de comunidades de práctica, grupos de personas con intereses comunes que entran en relaciones formalizadas con base en las metodologías de transferencia y gestión del conocimiento para la realización de un proyecto conjunto compartiendo repertorios y recursos. ${ }^{18}$

Con base en estos aprendizajes, el 3 de julio de 2009 se constituyó el Instituto Mesoamericano de Salud Pública (IMSP) como órgano técnico del SMSP y de la Red Virtual de Instituciones Académicas y de Investigación, con la participación del Proyecto Mesoamérica de Integración y Desarrollo, así como del Comisca. ${ }^{19-21}$ Las instituciones y organismos que integran el IMSP son: el Centro de Investigaciones y Estudios de la Salud de la Universidad Nacional Autónoma de Nicaragua; el Instituto Gorgas de Estudios de la Salud de Panamá; el Colegio de la Frontera Sur, Chiapas, México; la Facultad de Ciencias Médicas de la Universidad de San Carlos, Guatemala; el Instituto Nacional de Salud Pública de México; la Universidad de Costa Rica; la Facultad de Medicina de la Universidad de El Salvador; el Comisca; y el Proyecto Mesoamérica. El IMSP tiene como objetivos: a) contribuir al desarrollo de la gobernanza del SMSP, brindando apoyo técnico para el desarrollo de la Agenda Mesoamericana de Salud; b) fortalecer la capacidad técnica de los programas regionales de salud que formen parte del SMSP; y c) apoyar la integración de los programas regionales de salud a los sistemas nacionales de salud.*, , s\# El IMSP opera en los nueve estados de los estados del sur sureste de México, en todos los países de Centroamérica y en Colombia; está por integrarse la República Dominicana.

El IMSP opera tres programas regionales: el Programa de Formación y Actualización de Profesionales en Salud se enfoca en programas de doctorado y maestría y ha matriculado ocho estudiantes con el apoyo de becas de la Fundación Carlos Slim para los programas del Ins-

\footnotetext{
* Mesoamerican Health Initiative. Master Plan Maternal, Neonatal and Reproductive Health Working Group. October 2009. Documento de trabajo.

‡ Mesoamerican Health Initiative. The Master Plan: Recommendations for improving immunization in the most vulnerable populations of Mesoamerica Immunization. Working Group. October 2009. Documento de trabajo.

$\S$ Mesoamerican Health Initiative. Malaria Control to Elimination Master Plan. Technical Vector Group. October 2009. Documento de trabajo.

\# Mesoamerican Health Initiative. The Master Plan. Technical Nutrition Group. October 2009. Documento de trabajo
} 
tituto Nacional de Salud Pública de doctorado en Salud Pública y en Ciencias de Nutrición y para las maestrías en Ciencias y en Salud Pública. El programa de capacitación y desarrollo institucional apunta al desarrollo de competencias y habilidades en servidores públicos que se desempeñan en diferentes áreas de los sistemas de salud. El programa de apoyo técnico y gestión del conocimiento fomenta el establecimiento de una red de expertos apoyada en una plataforma de gestión del conocimiento, al servicio del SMSP.

Este artículo reúne los diversos estudios de diagnóstico y análisis estratégico que sirvieron de base para la planeación del IMSP. Presenta además resultados de evaluación preliminar del programa de capacitación a un año de su fundación. Sobre esta base se identifican los principales retos para su consolidación.

\section{Material y métodos}

La preparación del IMSP constó de tres estudios: un diagnóstico de capacidad para el desempeño de las once funciones esenciales en salud pública (reportado en otro artículo en ese mismo volumen); un diagnóstico de necesidades de fortalecimiento para la gestión del conocimiento en el grupo de trabajo del SMSP dedicado a malaria y dengue, y un análisis de contenido de necesidades de capacitación para el desempeño de los Planes Maestros para cada uno de los cuatro programas del SMSP.

Para el diagnóstico de necesidades para gestión del conocimiento se invitó a la autoaplicación de una encuesta por correo electrónico a 26 funcionarios y académicos que formaron parte del grupo de trabajo de malaria y dengue. La encuesta fue diseñada para que los equipos de trabajo liderados por los funcionarios identificara colectivamente sus capacidades, necesidades y oportunidades de mejora para la adquisición, análisis, adaptación y aplicación de la evidencia científica en la toma de decisiones. ${ }^{12}$ Se obtuvo la participación de ocho equipos (32\% tasa de respuesta) de México, Guatemala, Nicaragua, Costa Rica y Panamá, con un total de 23 participantes en sus equipos, que incluyó siete directores nacionales y coordinadores locales de programas en enfermedades transmitidas por vector, cuatro investigadores y 12 operativos.

El análisis de necesidades de capacitación se realizó en dos etapas: a) análisis de contenido de los cuatro programas de trabajo del SMSP, con identificación de las competencias en salud pública y administración de programas implicadas para el desempeño de cada uno de ellos; b) revisión documental de políticas, estrategias y programas regionales de la OMS/OPS relacionados con las intervenciones del SMSP, para identificar nece- sidades y / o recomendaciones para la capacitación del personal de salud en Mesoamérica. ${ }^{12,13}$ Sobre estas bases se realizó un mapeo de competencias a desarrollar en el personal de salud de los niveles estratégico, táctico y operativo, relacionados con la implementación de las intervenciones del SMSP. Asimismo, se identificaron instituciones de carácter académico y científico, públicas, sociales y privadas a las que se puede recurrir para el diseño e implementación de actividades de capacitación, de acuerdo con la temática identificada y su experiencia y ubicación en los países de la región. ${ }^{14}$

La evaluación de avance del programa de capacitación se realizó analizando información para tres cursos, 155 aspirantes y 91 egresados, toda ella reunida en el periodo de mayo de 2009 a abril de 2010. Se identificaron las competencias abordadas en cada curso, los objetivos de capacitación y las características del currículum. Con base en la información de la matrícula de participantes, se identificó el perfil funcionarial de los participantes para caracterizarlo ya fuera como estratégico, táctico u operativo. Los cursos fueron estandarizados calculando el total de horas-alumno brindadas, así como los costos por alumno. Se analizó la información según país, perfil funcionarial y tipo de institución beneficiada.

\section{Resultados}

\section{Fortalecimiento}

Los funcionarios encuestados poseen habilidades atribuidas a su formación y experiencia en los procesos de traducción del conocimiento científico, sin embargo, factores de la organización y del sector (estructura y procesos organizacionales, cultura política regional, lineamientos internacionales) dificultan la adopción de la traducción del conocimiento en la toma de decisiones. Esta situación se evidencia en la baja tasa de respuesta a la encuesta, en la que una de las primeras barreras fue el uso de internet y medios electrónico, la falta de espacios y tiempos en la organización para el diálogo, la discusión y la formulación de necesidades en equipo y la falta de iniciativas de autoevaluación y fortalecimiento sectorial. Por lo tanto, las iniciativas para incrementar las capacidades en traducción de evidencia científica deben estar precedidas y fortalecidas por programas de mejora en gestión del conocimiento, encaminados a fomentar el liderazgo y el desarrollo de una cultura organizacional en gestión de la información y la generación de nuevo conocimiento, a partir de la interacción formal (espacios de discusión virtuales o presenciales, seminarios, convenios, etc.) de demandantes y oferentes de evidencia científica, que fortalezca su utilización en la toma de decisiones en salud. 


\section{Capacitación}

Los grupos de trabajo identificaron grandes áreas temáticas en las cuales se requiere capacitación. Destacan áreas en salud pública como epidemiología, sistemas de salud, bioestadística, administración y gerencia en salud, así como promoción de la salud y participación social (cuadro I). Se identificaron los temas más frecuentes de capacitación según mención, por los grupos de trabajo en sus áreas sustantivas, así como para las áreas de gerencia de programas y de competencias para el cumplimiento de las funciones esenciales en salud pública.

El grupo de trabajo de nutrición se enfocó en las áreas de conocimiento de salud poblacional y de gerencia de programas de nutrición poblacional. Destacan necesidades de capacitación en:

- Diseño y ejecución de programas de educación para la salud nutricional

- Proceso de comunicación en salud

- Liderazgo en salud

- Ética en salud

- Multi y pluriculturalismo en nutrición y salud.

Este grupo de trabajo se concentró más en las competencias a desarrollar en el personal de salud del nivel táctico y operativo. Las principales competencias por desarrollar, identificadas para este grupo de trabajo según el nivel de los funcionarios a capacitar, fueron:

\section{Nivel estratégico}

- procesamiento deinformación, manejoeinterpretación de bases de datos, abogacía política y administración de recursos de programas de nutrición poblacional

- desarrollo, aplicación y promoción de procesos de comunicación efectiva para la dirección y coordinación de intervenciones en nutrición poblacional

- desarrollo, aplicación y promoción de habilidades de liderazgo en ejecución de intervenciones en nutrición poblacional

- valoración, aplicación y promoción del respeto a la diversidad y a la multiculturalidad

\section{Nivel táctico}

- obtención, análisis, interpretación e integración de conocimientos sobre nutrición poblacional

- movilización de recursos

- monitoreo y evaluación de programas

- coordinación de equipos de trabajo para programas de nutrición poblacional

- valoración, aplicación y promoción del respeto a la diversidad y a la multiculturalidad

\section{Nivel operativo}

- dirección de acciones de probada efectividad para la solución de problemas de salud de la población

- desarrollo de capacidad de comunicarse con empatía con la audiencia en nutrición

Cuadro I

Necesidades de capacitación, según grandes áreas temáticas y grupos de trabajo. Mesoamérica, 2009

Temas

Gerencia y dirección

Administración de servicios de salud (planeación, organización, dirección, supervisión y evaluación)

Promoción de la salud

Calidad de la atención

Bioestadística

Investigación participativa

Economía de la salud

Sistemas de información

Monitoreo y evaluación

Epidemiología y vigilancia epidemiológica

Participación social en salud

Liderazgo en salud
Grupos de trabajo

Nutrición y salud materna

Nutrición, salud materna y malaria Malaria y nutrición

Salud materna y nutrición

Malaria y vacunación

Malaria y nutrición

Malaria y nutrición

Vacunación, salud materna, nutrición y malaria

Nutrición, malaria y salud materna

Vacunación y malaria

Malaria, nutrición y salud materna

Nutrición y salud materna

Fuente: Cuestionario de necesidades de capacitación. IMSP, 2009 
- toma de decisiones basadas en evidencia durante la ejecución de intervenciones en nutrición poblacional

- valoración y respeto de la diversidad y de la multiculturalidad en la ejecución de acciones de nutrición poblacional

En el grupo de salud materna, reproductiva y neonatal destacan áreas relacionadas con la planificación familiar, la atención del parto y la atención neonatal. Entre la temática de capacitación destaca:

- anticoncepción posparto y posaborto

- anticoncepción para adolescentes

- costo-efectividad de métodos modernos de larga duración; implantes y DIU

- cuidado de la Emergencia Obstétrica (CEmO)

- buenas prácticas (intervenciones) para disminuir muerte materna

- prevención y manejo de asfixia neonatal: resucitación, referencia al hospital

- prevención y manejo de infecciones: parto limpio, uso de antibióticos, vacunas y cuidado piel-a-pie.

Entre las competencias a desarrollar según nivel destacan:

\section{Nivel estratégico}

- planeación, diseño, dirección y evaluación de programas de anticoncepción

- implementación de servicios de anticoncepción para adolescentes

- investigación operativa para definir la costoefectividad de introducir o reintroducir métodos modernos de larga duración

- planeación, diseño, dirección y evaluación de acciones de prevención y abatimiento de mortalidad materna

- planeación, diseño, dirección y evaluación de programas y acciones de atención neonatal

\section{Nivel táctico}

- supervisión y evaluación de programas de anticoncepción

- implementación de servicios de anticoncepción para adolescentes

- supervisión y evaluación de acciones de prevención y abatimiento de mortalidad materna

- supervisión y evaluación de programas y acciones de atención neonatal

\section{Nivel operativo}

- promoción de la anticoncepción

- realización, orientación y consejería sobre planificación familiar

- prescripción adecuada de métodos anticonceptivos

- identificación y aplicación de buenas prácticas en atención de parto y puerperio

- realización de cuidado de emergencia obstétrica

- identificación y aplicación de buenas prácticas en atención de casos de aborto

- aplicación de procedimientos de resucitación neonatal

- prevención de infecciones, atención de parto limpio, manejo adecuado de antibióticos, aplicación de cuidados y manejo de vacunas

El grupo de vectores hizo énfasis en la capacitación del personal comunitario para la gestión, la prevención, el control y la vigilancia de las enfermedades transmitidas por vector. Destacan los temas:

- análisis de salud y sistemas de información

- mecanismos de control de vectores que no repercutan en el medio ambiente

- gestión logística, financiera y de generación de recursos de los programas

- diagnóstico y tratamiento del paludismo y dengue apropiado y adecuado

- operación de redes para la prevención, la vigilancia y la detección del paludismo y dengue

- vigilancia de la farmacorresistencia de los medicamentos para el tratamiento de la malaria

- control de calidad en los laboratorios y uso de pruebas rápidas de diagnóstico

- investigación y desarrollo de tecnologías y herramientas para la prevención, la vigilancia y la detección

Entre las competencias a desarrollar para control de vectores según nivel, se identificaron:

\section{Nivel estratégico}

- planificación, organización, dirección y evaluación de políticas y estrategias de control de vectores sin comprometer al medio ambiente

- desarrollo de estrategias de gestión de recursos para los programas de prevención y control

- diseño y promoción de la integración de redes sociales e institucionales para la vigilancia y la detección de enfermedades transmitidas por vectores 
- promoción y establecimiento de alianzas estratégicas en la gestión de programas regionales y nacionales de prevención y control de enfermedades transmitidas por vector

- Establecimiento de mecanismos de mejoría y control de calidad en la prevención y control de vectores

\section{Nivel táctico}

- organización y conducción de acciones de control de vectores sin repercusiones negativas en el medio ambiente

- gestión de la ampliación de recursos para los programas de prevención y control

- análisis e interpretación de información para la toma de decisiones en la conducción de intervenciones

- organización y utilización de alianzas en la conducción y gestión de programas

- organización y conducción de acciones de vigilancia de la farmacorresistencia de los medicamentos para el tratamiento de la malaria

- desarrollo y aplicación de acciones de control de calidad en los laboratorios y manejo de pruebas rápidas de diagnóstico

\section{Nivel operativo}

- utilización de sistemas de información y sus productos para la toma de decisiones locales

- gestión local de la asignación de recursos para las acciones de prevención y control

- participación y coordinación de acciones de gestión de redes de servicios con participación de organismos de la sociedad civil y de la comunidad

- realización del diagnóstico y del tratamiento del paludismo y dengue apropiado y adecuado

- aplicación de medidas de control de vectores sin comprometer al medio ambiente

Para el grupo de trabajo de vacunación se identificaron los siguientes temas para capacitación:

- uso de la metodología participativa en el diseño, la ejecución, el seguimiento y la evaluación de programas de vacunación

- vigilancia epidemiológica y pruebas de laboratorio

- buen uso y control de la tecnología y monitoreo confiable y consistente

- indicadores regionales para programas de vacunación

- gestión de financiamiento externo para nuevas vacunas
En las necesidades de desarrollo de competencias se identificaron coincidencias de los grupos de trabajo para los niveles estratégico táctico y operativo. Entre las competencias que es necesario desarrollar y/o fortalecer en los funcionarios del nivel estratégico están: análisis de las necesidades y expectativas de salud de las poblaciones, evaluación de la situación de salud, priorización, diseño y dirección de intervenciones de manera eficiente con garantía en la calidad de la atención y acordes con las características culturales de las poblaciones regionales, nacionales y locales. En el ámbito táctico destacan competencias necesarias para organizar, dirigir, supervisar y evaluar intervenciones de salud nacional y local, al promover el establecimiento de redes de servicios y la participación sectorial y social en los programas y servicios de salud. En el ámbito operativo, las competencias necesarias por implementar son los programas e intervenciones locales, con redes de servicios y participación social (cuadro II).

Asimismo se identificaron instituciones académicas de la región que pueden dar respuesta a las necesidades de capacitación, de acuerdo con los temas. Aunque la relación no es exhaustiva, se puede apreciar la existencia de múltiples instituciones de carácter académico y científico, públicas, sociales y privadas, a las que se puede recurrir para el diseño e implementación de actividades de capacitación de acuerdo con la temática identificada y a su experiencia y ubicación en los países de la región (cuadro III).

\section{Evaluación del Programa de Capacitación y Desarrollo Institucional}

El Consejo Directivo (Codi) del IMSP emitió una convocatoria para las instituciones académicas integrantes del IMSP, con el objetivo de que presentaran ofertas de cursos cortos y diplomados de capacitación, con prioridad en el uso de la modalidad de educación a distancia, enfocado en temas y competencias por niveles identificados en el diagnóstico.

Se recibieron más de 30 propuestas de cursos y diplomados, con gran diversidad de temas y modalidades de enseñanza. Fueron revisadas por el secretariado del IMSP, que analizó la pertinencia de las mismas en relación con las necesidades y prioridades del SMSP. Se seleccionaron cursos ofertados por instituciones de cinco países (México, Guatemala, Costa Rica, Nicaragua y Panamá), de los cuales el Codi y la Fundación Carlos Slim (donante principal) aprobaron la impartición de dos cursos cortos y un diplomado ofertados por el Instituto Nacional de Salud Pública de México. El curso "Políticas de salud sobre bases científicas" tuvo como objetivos: a) desarrollar y aplicar herramientas para el aprovechamiento de la investigación para el desarrollo 


\section{Cuadro II

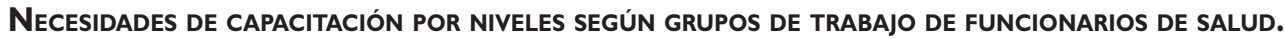 SMSP, MesoamÉrica, 2010}

\begin{tabular}{|c|c|c|c|}
\hline \multirow{2}{*}{ Grupo de trabajo } & \multicolumn{3}{|c|}{ Nivel } \\
\hline & Estratégico & Táctico & Operativo \\
\hline Nutrición & $\begin{array}{l}\text { Diseño y ejecución de progra- } \\
\text { mas de educación para la salud } \\
\text { nutricional } \\
\text { Desarrollar liderazgo y ética en } \\
\text { salud y nutrición }\end{array}$ & $\begin{array}{l}\text { Desarrollar el proceso de comunicación en } \\
\text { salud }\end{array}$ & $\begin{array}{l}\text { Identificar y aplicar multi y pluriculturalismo en } \\
\text { salud y nutrición }\end{array}$ \\
\hline $\begin{array}{l}\text { Salud materna, } \\
\text { neonatal y re- } \\
\text { productiva }\end{array}$ & $\begin{array}{l}\text { Análisis de costo-efectividad de } \\
\text { métodos modernos de larga du- } \\
\text { ración (implantes y DIU) } \\
\text { Diseño de intervenciones para } \\
\text { anticoncepción en adolescentes }\end{array}$ & $\begin{array}{l}\text { Promover acceso libre e informado a por lo } \\
\text { menos seis métodos de planificación familiar } \\
\text { modernos reversibles y referencia a AQV en } \\
\text { todos los centros de salud } \\
\text { Anticoncepción para adolescentes }\end{array}$ & $\begin{array}{l}\text { Servicios de anticoncepción posparto y pos- } \\
\text { aborto } \\
\text { Cuidado de la Emergencia Obstétrica (CEmO) } \\
\text { Manejo activo del tercer periodo del trabajo } \\
\text { de parto }\end{array}$ \\
\hline
\end{tabular}

Implementar buenas prácticas (intervenciones) para disminuir muerte materna

\begin{tabular}{ll}
\hline Aplicar metodologías y herra- \\
mientas en el diseño de proyectos \\
regionales y locales con desarrollo \\
sustentable para el control e \\
interrupción de la transmisión de \\
malaria y dengue
\end{tabular}

Identificar componentes biológicos, epidemiológicos, parasitológicos, entomológicos, geográficos y sociales en el control, prevención y eliminación de malaria y dengue

Aplicación de las buenas prácticas en el diseño, ejecución y evaluación de las estrategias para el control y eliminación de malaria y dengue Realizar una vigilancia epidemiológica confiable (parasitológica y entomológica) en el control de enfermedades trasmitidas por malaria y dengue

Supervisar y evaluar programas con enfoque de vulnerabilidad

Monitoreo confiable y consistente

Vigilancia epidemiológica y pruebas de laboratorio

Uso de la metodología participativa en el diseño, la ejecución, el seguimiento y la evaluación de programas de vacunación
Generar estrategias que promuevan la participación de la comunidad para la prevención y control de dengue y malaria

Desarrollar las capacidades técnicas y de toma de decisiones necesarias en los programas de vigilancia, prevención y control de malaria y dengue

Implementar campañas de salud con enfoque de interculturalidad, migración y equidad Identificar y aplicar buenas prácticas de costobeneficio en la implementación de esquemas de vacunación

Buen uso de tecnología en vacunación

Uso de la metodología participativa en la ejecución, el seguimiento y la evaluación de actividades de vacunación

Fuente: Cuestionario de necesidades de capacitación. IMSP, 2009.

de programas regionales de alta prioridad en salud; b) promover la utilización de la investigación y desarrollar la capacidad para adquirir, analizar, adaptar y aplicar resultados de investigación en la toma de decisiones; y c) fortalecer las habilidades de liderazgo para desarrollar una cultura organizacional que promueva la utilización de los resultados de investigación en la toma de decisiones. El curso contó con 35 aspirantes, de los que se aceptó a 23 alumnos de siete países de la región.

$\mathrm{El}$ "Curso regional de gestión y liderazgo de políticas y programas de salud" tuvo como objetivo desarro- llar la capacidad para aplicar herramientas que faciliten la formulación, la implementación, la evaluación y la gestión financiera de programas regionales en salud pública. De esta manera, contribuye al fortalecimiento de la gobernanza y a la aplicación de un enfoque global en el análisis de la problemática de salud y al diseño de intervenciones de carácter regional y multilateral. Solicitaron su ingreso más de 80 aspirantes, ingresaron 50 alumnos de siete países de la región.

El "Diplomado en salud global" tuvo como objetivo desarrollar la capacidad para analizar, dirigir y respon- 


\section{Cuadro III}

InStituciones de Mesoamérica QUe pUeden satisfacer NECESIDADES DE CAPACITACIÓN SEGÚN TEMA. SMSP, MeSOAMÉRICA, 2009

\author{
Necesidad de \\ capacitación en \\ Institución que puede satisfacer esta necesidad
}

\begin{tabular}{|c|c|}
\hline $\begin{array}{l}\text { Epidemiología } \\
\text { y vigilancia } \\
\text { epidemiológica }\end{array}$ & $\begin{array}{l}\text { Instituto Nacional de Salud Púbica (México) } \\
\text { Universidad de Panamá (Escuela de SP) } \\
\text { Centro de Investigación y Estudios en Salud (Nicaragua) } \\
\text { Universidad de San Carlos de Guatemala }\end{array}$ \\
\hline Bioestadística & $\begin{array}{l}\text { Instituto Nacional de Salud Púbica (México) } \\
\text { Escuela de Salud Pública de la Universidad de Panamá } \\
\text { Centro de Investigación y Estudios en Salud (Nicaragua) } \\
\text { Escuela de Estadística de la Universidad de Costa Rica } \\
\text { Centro Centroamericano de Población (Costa Rica) }\end{array}$ \\
\hline Sistemas de salud & $\begin{array}{l}\text { Instituto Nacional de Salud Púbica (México) } \\
\text { Centro de Investigación y Estudios en Salud (Nicaragua) }\end{array}$ \\
\hline $\begin{array}{l}\text { Administración } \\
\text { de servicios de } \\
\text { salud (planeación, } \\
\text { organización, } \\
\text { supervisión) }\end{array}$ & $\begin{array}{l}\text { Instituto Nacional de Salud Púbica (México) } \\
\text { Centro de Investigación y Estudios en Salud (Nicaragua) } \\
\text { Universidad Estatal a Distancia (Costa Rica) } \\
\text { Universidad de Ciencias Médicas } \\
\text { Instituto Centroamericano de Salud Pública } \\
\text { Universidad de San Carlos de Guatemala }\end{array}$ \\
\hline $\begin{array}{l}\text { Gerencia y } \\
\text { dirección }\end{array}$ & $\begin{array}{l}\text { Instituto Nacional de Salud Púbica (México) } \\
\text { Instituto Nacional de Administración Pública (México) } \\
\text { Centro de Investigación y Estudios en Salud (Nicaragua) } \\
\text { Universidad Nacional y Universidad Latina (Panamá) } \\
\text { Universidad Interamericana (Panamá) }\end{array}$ \\
\hline Liderazgo en salud & Instituto Nacional de Salud Púbica (México) \\
\hline ón de la & $\begin{array}{l}\text { Instituto Nacional de Salud Púbica (México) } \\
\text { Universidad de Panamá } \\
\text { Universidad de las Américas (Panamá) } \\
\text { Universidad de Costa Rica }\end{array}$ \\
\hline $\begin{array}{l}\text { Participación social } \\
\text { en salud }\end{array}$ & $\begin{array}{l}\text { Instituto Nacional de Salud Púbica (México) } \\
\text { Universidad de Costa Rica } \\
\text { Universidad de Panamá } \\
\text { Universidad de las Américas (Panamá) }\end{array}$ \\
\hline $\begin{array}{l}\text { Investigación } \\
\text { participativa }\end{array}$ & $\begin{array}{l}\text { Instituto Nacional de Salud Púbica (México) } \\
\text { Universidad de Costa Rica } \\
\text { Universidad Estatal a Distancia (Costa Rica) } \\
\text { Universidad Nacional (Costa Rica) } \\
\text { Instituto Tecnológico de Costa Rica } \\
\text { Universidad de Panamá } \\
\text { UDELAS (Panamá) }\end{array}$ \\
\hline $\begin{array}{l}\text { Economía de la } \\
\text { salud }\end{array}$ & $\begin{array}{l}\text { Instituto Nacional de Salud Púbica (México) } \\
\text { Universidad de Costa Rica } \\
\text { Instituto Tecnológico de Costa Rica } \\
\text { Centro de Investigación y Estudios en Salud (Nicaragua) } \\
\text { Universidad de Panamá }\end{array}$ \\
\hline $\begin{array}{l}\text { Sistemas de } \\
\text { información }\end{array}$ & $\begin{array}{l}\text { Instituto Nacional de Salud Púbica (México) } \\
\text { Instituto Tecnológico de Costa Rica } \\
\text { Instituto Gorgas (Panamá) }\end{array}$ \\
\hline $\begin{array}{l}\text { Calidad de la } \\
\text { atención a la salud }\end{array}$ & $\begin{array}{l}\text { Instituto Nacional de Salud Púbica (México) } \\
\text { Instituto Nacional de Administración Pública (México) } \\
\text { Centro de Investigación y Estudios en Salud (Nicaragua) } \\
\text { Universidad de Costa Rica } \\
\text { Universidad Nacional de El Salvador } \\
\text { Instituto Centroamericano de Salud Pública } \\
\text { Universidad de San Carlos de Guatemala }\end{array}$ \\
\hline $\begin{array}{l}\text { Monitoreo y } \\
\text { evaluación }\end{array}$ & $\begin{array}{l}\text { Instituto Nacional de Salud Púbica (México) } \\
\text { Universidad de Costa Rica } \\
\text { Instituto Tecnológico de Costa Rica } \\
\text { Instituto Gorgas (Panamá) }\end{array}$ \\
\hline
\end{tabular}

Fuente: Cuestionario de necesidades de capacitación. IMSP, 2009 der a los retos de la salud pública y sus necesidades en el contexto de un mundo cada vez más globalizado e interconectado. A este diplomado solicitaron su ingreso 35 aspirantes, y fueron aceptados 18 alumnos de cinco países de la región.

En total, se capacitó a 91 funcionarios de la región, de los cuales $22 \%$ corresponde a funcionarios del nivel estratégico, $60 \%$ al nivel táctico y $18 \%$ al nivel operativo.

De los funcionarios capacitados, según país, la tercera parte fueron originarios de México, seguidos en frecuencia por Panamá, Colombia y El Salvador. Según nivel gerencial, México y Panamá fueron los países con mayor número de capacitados de nivel estratégico. De nivel táctico los países que más funcionarios capacitaron fueron México y El Salvador. En el nivel operativo predominaron funcionarios de Colombia y México. De acuerdo con la institución de origen, una tercera parte correspondió a funcionarios de ministerios de salud, seguidos en frecuencia por funcionarios de instituciones académicas, seguidos por instituciones de seguridad social.

De acuerdo con nivel gerencial e institución de origen, en el nivel estratégico predominaron funcionarios de ministerios de salud; en el nivel táctico predominaron funcionarios de instituciones académicas. En cuanto a la participación de funcionarios de nivel operativo, más de la mitad provenía de ministerios de salud e instituciones académicas.

Según participación en grupos de trabajo (cuadro III), aproximadamente $40 \%$ correspondió a funcionarios relacionados con salud materna, reproductiva y neonatal. El restante $60 \%$ correspondió de manera similar cercana a los grupos de trabajo de malaria-dengue, nutrición y vacunación (20\% cada uno). Por grupo de trabajo y nivel gerencial, para el nivel estratégico táctico operativo predominaron funcionarios relacionados con salud materna, reproductiva y neonatal, así como del grupo de malaria y dengue. En total se impartieron 5800 horas/ persona de capacitación, más de la mitad de las cuales la recibieron funcionarios del nivel táctico, seguidos del nivel operativo y en menor frecuencia del nivel estratégico.

\section{Discusión}

En su primer año de trabajo, el IMSP logró importantes logros para el diagnóstico de las necesidades de fortalecimiento de las competencias de los funcionarios y trabajadores de salud en la región. Gracias al apoyo del Instituto Carlos Slim de la salud se consiguió un rápido despegue, demostrado por la alta demanda de participantes a los cursos y el diplomado. Se estableció asimismo un proceso de convocatorias y selección que resultó 


\section{Cuadro IV}

FUNCIONARIOS MESOAMERICANOS POR NIVEL GERENCIAL, FORMADOS Y CAPACITADOS POR EL IMSP, SEGÚN PAís, INSTITUCIÓN Y ÁREA de INTERVENCIÓN DEL SMSP. MESOAMÉRICA, 2009

\begin{tabular}{|c|c|c|c|c|}
\hline \multirow{3}{*}{ Concepto } & \multicolumn{4}{|c|}{ Capacitación* } \\
\hline & \multicolumn{4}{|c|}{ Nivel } \\
\hline & Estratégico & Táctico & Operativo & Total \\
\hline \multicolumn{5}{|l|}{ País } \\
\hline México & 8 & 16 & 10 & 34 \\
\hline Guatemala & & 4 & I & 5 \\
\hline Honduras & 1 & 6 & & 7 \\
\hline El Salvador & I & 10 & & 11 \\
\hline Nicaragua & 3 & 3 & & 6 \\
\hline Costa Rica & & 3 & & 3 \\
\hline Panamá & 7 & 6 & & 13 \\
\hline Colombia & & 7 & 5 & 12 \\
\hline Total & $\begin{array}{c}20 \\
(920 \mathrm{~h})^{*}\end{array}$ & $\begin{array}{c}55 \\
(3760 h) *\end{array}$ & $\begin{array}{c}16 \\
(1 \mid 20 h)^{*}\end{array}$ & $\begin{array}{c}91 \\
(5800) *\end{array}$ \\
\hline
\end{tabular}

Institución

\begin{tabular}{|c|c|c|c|c|}
\hline Ministerio de salud & 14 & 18 & 5 & 37 \\
\hline Seguridad Social & 3 & 10 & 3 & 16 \\
\hline Académica & 1 & 25 & 6 & 32 \\
\hline Privada & 2 & 2 & 2 & 6 \\
\hline Total & $\begin{array}{c}20 \\
(920 \mathrm{~h}) *\end{array}$ & $\begin{array}{c}55 \\
(3760 h)^{*}\end{array}$ & $\begin{array}{c}16 \\
(1120 h)^{*}\end{array}$ & $\begin{array}{c}91 \\
(5800)^{*}\end{array}$ \\
\hline
\end{tabular}

Área de intervención SMSP

\begin{tabular}{|c|c|c|c|c|}
\hline Nutrición & 3 & 9 & 2 & 14 \\
\hline Salud Materna & 9 & 24 & II & 44 \\
\hline Vacunación & 4 & 10 & I & 15 \\
\hline Malaria-dengue & 4 & 12 & 2 & 18 \\
\hline Total & $\begin{array}{c}20 \\
(920 \mathrm{~h})^{*}\end{array}$ & $\begin{array}{c}55 \\
(3760 \mathrm{~h})^{*}\end{array}$ & $\begin{array}{c}16 \\
(1120 \mathrm{~h})^{*}\end{array}$ & $\begin{array}{c}91 \\
(5800)^{*}\end{array}$ \\
\hline
\end{tabular}

Fuente: Informe final de cursos y diplomados 2009, IMSP

* Horas/persona:Total 5800 horas

exitoso, si bien el proceso de toma de decisiones fue en ocasiones poco claro, debido al ajuste en las expectativas entre los diversos actores. Las competencias identificadas permitirán el desarrollo curricular en el corto plazo, pues seguramente el diagnóstico cambiará pronto en función de las experiencias obtenidas con la propia capacitación y en la implementación de los programas.

EL IMSP fue puesto a prueba con base en cursos previamente diseñados por el INSP o que fueron diseñados en su totalidad. Una siguiente fase (en proceso) incluye la participación de otras instituciones del Codi en la impartición de cursos. En una próxima fase habrá de ampliarse la docencia a instituciones de la región, así como fuera de ella, siempre buscando el enfoque regional como sello característico y como principal valor agregado. Así, el IMSP está convirtiéndose en un mecanismo para promover el desarrollo curricular regional, con vistas a fortalecer las competencias del personal en una dimensión complementaria al esfuerzo nacional.

El IMSP tiene también un futuro importante en la gestión del conocimiento regional con base en la puesta en práctica de diferentes herramientas, como la síntesis de literatura sobre intervenciones exitosas, la organización de comunidades de práctica y la aplicación de técnicas para la vinculación entre la experiencia de los funcionarios y la investigación científica. Tanto la capacitación como la gestión del conocimiento están a disposición de las diversas iniciativas que componen al SMSP. 


\section{Conclusiones}

El IMSP responde a las exigencias de salud global y a las relaciones de colaboración entre instituciones académicas y gubernamentales en los problemas regionales de salud, logrando una concentración regional efectiva. El IMSP, como órgano técnico del SMSP, integra la colaboración de organismos públicos y privados para dar legitimidad y normatividad a las aportaciones a los sistemas de salud de la región, y promueve la participación de los organismos de la sociedad civil en todos los niveles. Así, juntos pueden enfrentar la problemática de la salud pública en Mesoamérica.

El IMSP puede desarrollar otras líneas de investigación en sistemas de salud de forma paralela, para ampliar el enfoque en otros problemas de salud pública, fortaleciendo los Objetivos del Milenio 3, 4 y 5.

\section{Agradecimientos}

Se agradece al Instituto Carlos Slim de la Salud y a la Secretaría de Salud de México por el apoyo financiero para las actividades del IMSP, así como a todos los integrantes de los grupos de trabajo del SMSP por su colaboración en el diagnóstico de capacidades.

Declaración de conflicto de intereses: Los autores declararon no tener conflicto de intereses.

\section{Referencias}

I. Nigenda G. La década de los recursos humanos en salud: retos para su investigación y planeación Salud Pública de México [en línea] 2007, 49 [Consultado 2010 agosto 9]. Disponible en: <http://redalyc.uaemex.mx/ redalyc/src/inicio/ArtPdfRed.jsp? iCve=I0649022> ISSN 0036-3634. 2. Sepúlveda J, Bustreo F, Tapia R, Rivera J, Lozano R, Oláiz G, et al. Improvement of child survival in Mexico: the diagonal approach. Lancet 368;955I:2017-2027.

3. Organización Panamericana de la Salud. Desarrollo del Talento Humano en Salud. Organización Panamericana de la Salud, Colombia [Consultado 2010 agosto 9] Disponible en: http://new.paho.org/col.

4. Allotey P, Reidpath DD, Ghalib H, Pagnoni F, Skelly WC. Efficacious, effective, and embedded interventions: Implementation research in infectious disease control. BMC Public Health 2008;8:343.

5. Hayward R. Informing health choices. Centre for Health Evidence Departments of Medicine and Public Health Sciences. University of Alberta, 2003 [Consultado 201 I junio I2] Disponible en: http://www. cche.net/about/files/Informing_Health_Choices_Oxford.pdf.
6. Hanney SR, González-Block MA. Evidence-informed health policy: are we beginning to get there at last? SUPPORT Tools for evidence-informed health Policymaking (STP). Health Res Policy Syst 2009;7(I):Editorial. 7. Lavis JN, Oxman A, Souza N, Lewin S, Gruen R, Fretheim A, et al. SUPPORT Tools for evidence-informed health Policymaking (STP). Health Res Policy Syst 2009;7(I):II [Consultado 20 II junio I2]. Disponible en: http://www.health-policy-systems.com/content/pdf//478-4505-7SI-II.pdf.

8. Oxman A, et al. A framework for mandatory impact evaluation to ensure well informed public policy decisions. Lancet 2010;375:433-524.

9. Graham I, Logan J, Harrison MB, Straus SE, Tetro J, Caswell W, Robinson N. Lost in translation:Time for a Map? The Journal of continuing Education in the Health Professions 2006;26(I).

10. National Cancer Institute. Greater Than the Sum: Systems Thinking in Tobacco Control. Capítulo 7. What We Know. Tobacco Control Monograph No. 18. Bethesda, MD: US Department of Health and Human Services, National Institutes of Health, National Cancer Institute. NIH Pub. núm. 06-6085, abril 2007 [Consultado 201 I junio 12]. Disponible en: http://dccps.cancer.gov/TCRB/monographs//8/m I8_complete.pdf. II. Durcikova A, Everard A. Managing the Corporate Zoo:A Knowledge Management Perspective. Electronic Journal of Knowledge Management 2004;2(I):23-28. Disponible en: http://www.ejkm.com/volume-2/v2il / v2-il-art2-durcikova.pdf.

I2. González-Block MA, Mercado FJ, Ochoa H, Rivera H, Idrovo JI. Utilización de investigación por gestores de salud. Desarrollo y validación de una herramienta de autodiagnóstico para países de habla hispana. Salud Publica Mex 2008;50(6).

13. Supporting Policy relevant Reviews and Trials Support 2010. Diversas herramientas [Consultado 20II junio I2]. Disponible en: http://www.support-collaboration.org/index.htm

14. National Collaborating Centre for Methods and Tools NCCMT. Introduction to Evidence-Informed Decision Making. 2010 [Consultado 201 I junio 12]. Disponible en: http://learning.nccmt.ca/en/ index.php

15. González-Block MA, Mercado FJ, Ochoa H, Rivera H, Idrovo AJ. Utilización de la investigación por gestores de salud: desarrollo y validación de una herramienta de autodiagnóstico. Salud Publica Mex 2008:50:490-497.

16. González-Block MA, Mercado FJ, Ochoa H, Rivera H, Idrovo AJ. Utilización de la investigación por gestores de salud en México: diagnóstico de la capacidad y propuestas de fortalecimiento. Salud Publica Mex 2008;50:498-507.

17. Centro de Investigación en Sistemas de Salud. Investigación para el Desarrollo de los Sistemas Estatales de Salud. Plataforma de Gestión del Conocimiento, 2010. Sitio de acceso restringido a usuarios habilitados [Consultado $201 \mathrm{I}$ junio 12]. Disponible en: https://sites.google.com/a/ imesoamericano.org/plataforma/

18. Wenger E. Communities of Practice: Learning, Meaning, and Identity. Cambridge: Cambridge University Press, 1998.

19. Plan Integral para el Desarrollo de un Sistema Mesoamericano de Salud Pública. Disponible en: http//www.imesoamericano.org/docs/pdf/ Mesoamerica_20febG.pdf

20. Misión del Instituto Mesoamericano de Salud Pública. Disponible en: http://www.imesoamericano.org/dodo.php?ident=mision 21. Instituto Mesoamericano de Salud Pública. Planeación estratégica y Desarrollo. Propuesta de Financiamiento. México: CISS-INSP, 2009. 